\title{
Superheavy Element - laying the grounds for the landing on the island of stability
}

\author{
Dieter Ackermann ${ }^{1}$ \\ GSI Helmholtzzentrum für Schwerionenforschung $\mathrm{GmbH}$ \\ Planckstr.!, D-64921 Darmstadt, Germany \\ E-mail: d.ackermann@gsi.de
}

The search for the next closed proton and neutron shells beyond ${ }^{208} \mathrm{~Pb}$ has yielded a number of exciting results in terms of the synthesis of new elements at the upper end of the charts of nuclides, in a region of exotic high- $Z$ nuclear matter. In particular, the results obtained at the Flerov Laboratory of Nuclear Reactions (FLNR) for a rich number of decay patterns for ${ }^{48} \mathrm{Ca}$ induced reactions on actinide targets have by now been confirmed for reactions on ${ }^{238} \mathrm{U}$ and ${ }^{244} \mathrm{Pu}$ at GSI, and on ${ }^{242} \mathrm{Pu}$ at LBNL. These superheavy elements (SHE), however, are a nuclear structure phenomenon. They owe their existence to shell effects, an energy contribution of quantum mechanical origin to the nuclear potential, without which they would not be bound. Experimental activities in this field, apart from attempts to directly synthesise new elements, have to investigate reaction mechanism studies and, in particular, they have to pursue nuclear structure investigations to study the development of single particle levels towards the expected gap for the proton and neutron shell closure in the region of the spherical SHE. Chemistry studies of SHE provide additional input on chemical properties and the $\mathrm{Z}$ assignment. Precision mass measurements yield an important input in terms of nuclear binding energies for theoretical models. In recent years the development of efficient experimental set-ups, including separators and advanced particle and photon detection arrangements, allowed for more detailed nuclear structure studies for nuclei at and beyond $Z=100$. Among the most interesting features is the observation of $K$-isomeric states. Experimentally about 13 cases have been identified in the region of $Z>96$. $K$-isomers or indications of their existence have been found for almost all even$Z$ elements in the region $Z=100$ to 110 . We could recently establish and/or confirm such states in the even-even isotopes ${ }^{252,254}$ No. The heaviest nucleus where such a state was found is ${ }^{270} \mathrm{Ds}$ with $Z=110$ as we reported in 2001. Those nuclear structure studies lay out the grounds for a detailed understanding of these heavy and high- $Z$ nuclear systems, and contribute at the same time valuable information to the preparation of strategies to successfully continue the hunt for the localisation of the next spherical proton and neutron shells beyond ${ }^{208} \mathrm{~Pb}$.

XLVIII International Winter Meeting on Nuclear Physics - BORMIO2010

Bormio, Italy

January 25-29 2010

\footnotetext{
$1 \quad$ Speaker
} 


\section{Introduction}

Four decades after the first prediction of the "island of stability" of SHE in the late sixties of the last century [1] its localization seems almost in reach with experimental indications up to a $\mathrm{Z}$ of 118 [2,3]. The two approaches cold and hot fusion, have yielded the synthesis of a large number of isotopes in the region of highest $\mathrm{Z}$ and $\mathrm{A}$. Their decay properties are to some extent consistent with most model predictions. As an example the results of a microscopicmacroscopic calculation [4] is shown in terms of ground-state shell correction energies in the same figure. At GSI cold fusion reactions have been employed to produce elements up to $Z=112$ [2] whereas the heaviest nucleus for this approach has been synthesized at RIKEN in the reaction ${ }^{70} \mathrm{Zn}+{ }^{209} \mathrm{Bi}[5]$. In ${ }^{48} \mathrm{Ca}$ induced reactions on actinide target nuclei decay patterns have been observed at Dubna which were assigned to the production of nuclei spanning the more neutron rich area from ${ }^{266} \mathrm{Rf}$ to ${ }^{294} 118$. Some of these results for decay chains assigned to isotopes of the elements $Z=112$ and 114 have been confirmed independently at the velocity filter SHIP at GSI [6], at the Berkeley gas-filled separator BGS at LBNL [7], and most recently for the reaction ${ }^{48} \mathrm{Ca}$ on ${ }^{244} \mathrm{Pu}$ at the gas-filled separator TASCA at GSI [8]. Beyond the successful synthesis of heavy nuclei, the high beam intensities nowadays available, together with the advanced particle and $\gamma$ detector set-ups allow for detailed nuclear structure investigations for heavy and super heavy nuclei like ${ }^{252,254}$ No $[9,10]$ and ${ }^{270}$ Ds [11] which will be discussed in this paper. In addition fast SHE chemistry, most recently performed after preseparation with the gas-filled separator TASCA, and precision mass measurements in the penning trap system SHIPTRAP complete the experimental means available at GSI. As one of the highlights in the field of heavy and superheavy nuclei the masses of the nobelium isotopes ${ }^{252,253,254}$ No could be measured with high precision at SHIPTRAP recently [12]. All these investigations call for a constant development of higher beam intensities due to the ever lower production cross sections.

\section{Superheavy Elements - Synthesis}

The identification of superheavy elements in heavy ion fusion reactions is based on two major ingredients: the separation of the fusion products in flight from the beam particles and the identification of the products via ER- $\alpha$ correlations. The velocity filter SHIP at GSI e.g. provides separation, using the velocity difference between the faster beam and the slower fusion products in the fashion of a classical Wien-filter, via the comparison of crossed E- and B-fields, in a separated field configuration. The particles passing the velocity filter are then implanted into a position sensitive silicon strip detector set-up where position, time and energy of the fusion products and subsequent decays by $\alpha$ emission and spontaneous fission are recorded. The $\mathrm{Z}$ - and A-identification of the starting point of those decay chains is unambiguously provided by the connection to known $\alpha$ emitters at the end of the decay sequence.

For the reactions employing fusion with ${ }^{208} \mathrm{~Pb}$ and ${ }^{209} \mathrm{Bi}$ as target, no deviation from the exponential decrease of the maximum cross section with increasing $\mathrm{Z}$ has been observed. The Dubna results for ${ }^{48} \mathrm{Ca}$ induced reactions on actinide targets, however, show cross-section values which surprisingly remain rather high and constant around and above the 1-pb-level for $\mathrm{Z}$ up to 118 [2]. In order to confirm these tempting results with the promising perspectives of relatively high production yields we chose as a first reaction ${ }^{48} \mathrm{Ca}+{ }^{238} \mathrm{U}$ with ${ }^{286} 112$ as compound nucleus [6]. The experiment was performed in three parts with a total of 88 days in the period from 2005 to 2007. The observed decay properties are in good agreement with the data obtained earlier at 
the FLNR [13,14]. We obtained a cross-section value of $0.72_{-0.35}^{+0.58} \mathrm{pb}$ as compared to the $2.5_{-1.1}^{+1.8} \mathrm{pb}$ reported in ref. [13]. Apart from the overlapping error bars, this slight discrepancy can be due to systematic deviations (see discussion in ref. [6]). In addition to our confirmation there was a chemical study of the reaction ${ }^{48} \mathrm{Ca}+{ }^{244} \mathrm{Pu}$ which observed also consistent decay patterns [15]. Most recently the same reaction was used at the gas-filled separator TASCA at GSI to successfully reproduce the same decay patterns assigned to isotopes of element $\mathrm{Z}=114$ [8] by Yu.Ts. Oganessian et al. [2].

Those results produced consistently in different laboratories and at different types of separators are a qualitative step forward towards a final $\mathrm{A}$ and $\mathrm{Z}$ assignment which is despite the impressive body of data still not final. Additional means are needed like chemistry where first impressive results have already been obtained and mass measurements.

To reach nuclei beyond $\mathrm{Z}=118$ heavier actinide targets are needed. To this end a program to use ${ }^{248} \mathrm{Cm}$ as target material at SHIP is presently being pursued.

\section{Superheavy Elements - Nuclear Structure}

There are two complementary approaches to explore the structure of heavy and superheavy nuclei. A comprehensive comparison review of nuclear structure investigations for heavy actinide and transactinide nuclei has been published by M. Leino and F.P. Heßberger [16]. Inbeam studies using a $\gamma$-spectroscopy set-up at the target position yield access to the high spin region of nuclear excitation in heavy ion collisions. They are, however, usually hampered by the high background rate from the close by and unshielded target what limits the beam current to moderate intensities. Decay spectroscopy after beam separation yields access to low lying states populated by $\alpha$-decay or below isomeric states only. It is, however, almost background free due to separation and decay coincidences. Moreover, the full intensities of high current accelerators can be used. The combination of the UNILAC accelerator with the velocity filter SHIP and the decay spectroscopy in its focal plane is among the most efficient setups for studies of this type worldwide. A similar configuration of particle and photon detectors called TASISPEC [17] is presently being set up at the focal plane of TASCA.

\subsection{Decay Spectroscopy after Separation}

The detailed understanding of nuclear structure and its development in the vicinity of closed shells, in regions of deformation and towards heavier masses and higher $\mathrm{Z}$ is a necessary ingredient for a successful progress in the synthesis of new heavy elements. The possible trends in single particle levels are the most sensitive probe for the formation of low level density, and eventually the appearance of shell gaps and regions of (shell-) stabilized nuclei. Decay spectroscopy of $\alpha$-emitters stopped after separation is a powerful tool to study their daughter products or isomeric states via $\alpha$ fine structure or $\alpha-\gamma$ spectroscopy by ER- $\alpha$ or ER- $\alpha-\gamma$ coincidence measurements. Here the fusion reaction products are after separation implanted into a solid state ("stop") detector for the residue and $\alpha$ detection, which is combined with a high resolving $\gamma$-ray (Ge-) detector array. A typical set-up scheme is shown in Fig. 1 and a review of the field can be found on ref. [9]. This method is very clean as compared to in-beam studies because of the effective shielding from target background due to its spatial separation and the effective cleaning by the ER- $\alpha$ coincidence technique. It is highly efficient because of the favourable close geometry of the $\alpha$ and $\gamma$ detectors and the very well localized stopped $\gamma$-ray source that the implantation spot of the ER is forming on the stop detector. The latter has the further advantage of the absence of any $\gamma$-ray Doppler shift or broadening which yields, with a 
moderate crystal size and a moderate granularity of the Ge-detector, a nevertheless high efficiency $\varepsilon$. In case of the SHIP set-up we achieve an $\varepsilon$ of up to $\approx 15 \%$.

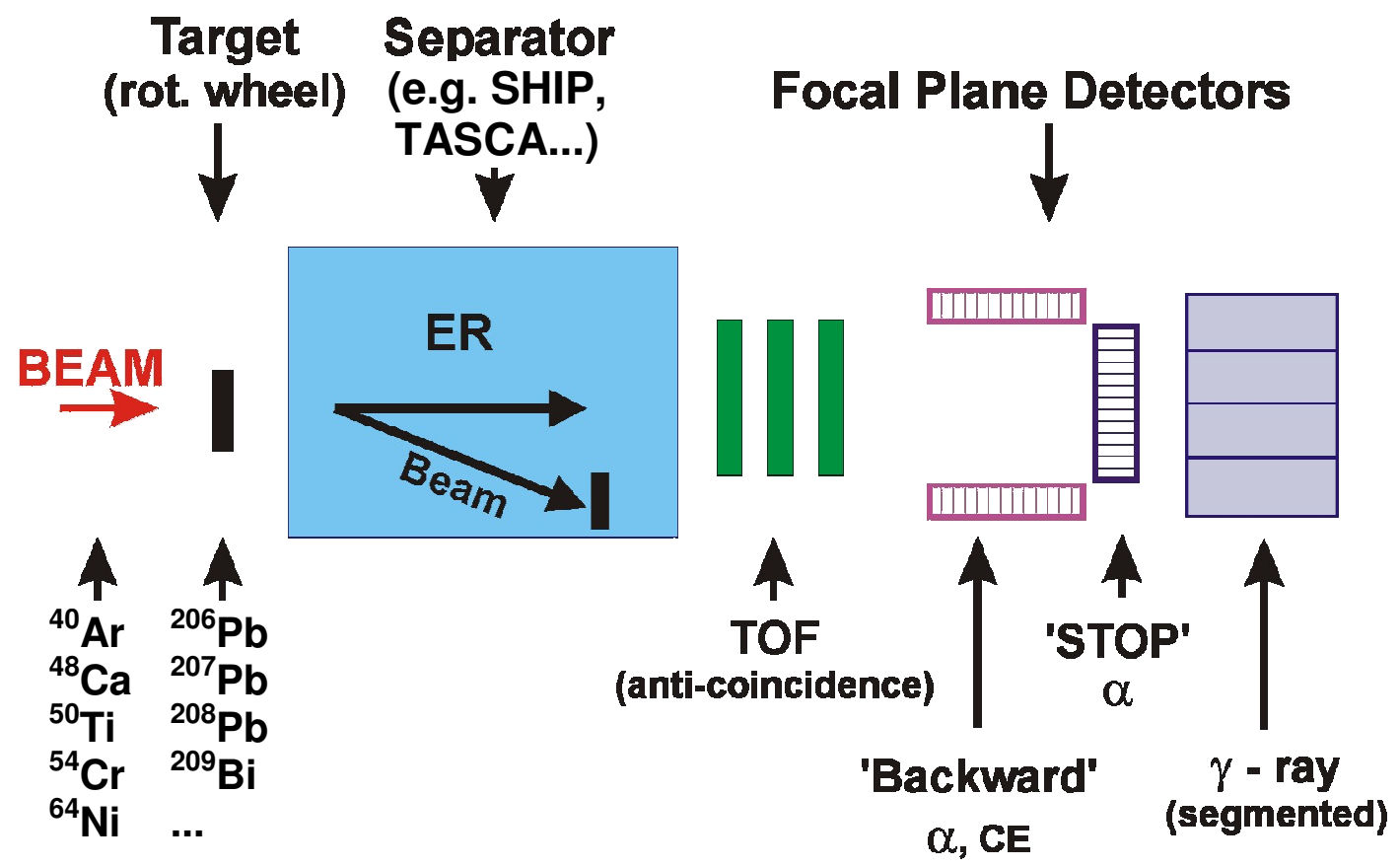

FIGURE 1. Scheme of a typical decay spectroscopy set-up for particle, $\gamma$-ray and X-ray detection.

\section{2(K)-Isomers}

Among the most interesting features is the observation of $K$-isomeric states. The $K$-quantum number is the projection of the total angular momentum $J$ on the symmetry axis of an axially deformed nucleus. In prolate deformed nuclei isomeric states are formed when their decay requires a large change in $K$ value. Transition are called " $K$-forbidden" when the change in the $K$ value is larger then the transition multipole order $\lambda$ with the degree of forbiddenness defined as $v=\Delta K-\lambda$ (see e.g. $[19,20])$.

$\mathrm{Xu}$ et al., employing configuration constrained potential energy surface calculations (PES), predict high $K$-isomers to be a general feature of prolate deformed nuclei in the region for $Z>100$ in terms of nucleon pair breaking into multi-quasiparticle excitations at low excitation energies $(\approx 1-2 \mathrm{MeV})$ for even-even isotopes [21]. In fact, experimentally about 13 cases have been identified in the region of $Z>96$ as shown in Fig. 2. A table of $K$-isomers known in eveneven isotopes in that region is given in ref. [18]. $K$-isomers or indications of their existence have been found for almost all even- $Z$ elements in the region $Z=100$ to 110 . We could recently establish and/or confirm such states in the even-even isotopes ${ }^{252,254}$ No [22]. The heaviest nucleus where such a state was found is ${ }^{270} \mathrm{Ds}$ as reported by our group in 2001 [11]. In the same work we noticed the possibility of an isomeric state in the ${ }^{270}$ Ds daughter nucleus ${ }^{266} \mathrm{Hs}$ (see 3.2.2.). For $Z=106$ the search was up to now not successful. However, $K$-isomers were found also in even-odd and odd-even isotopes in this region like e.g. ${ }^{251}$ No [22], ${ }^{253} \mathrm{No}$ [23], ${ }^{255}$ No [24], and ${ }^{255} \operatorname{Lr}[25,26]$. 


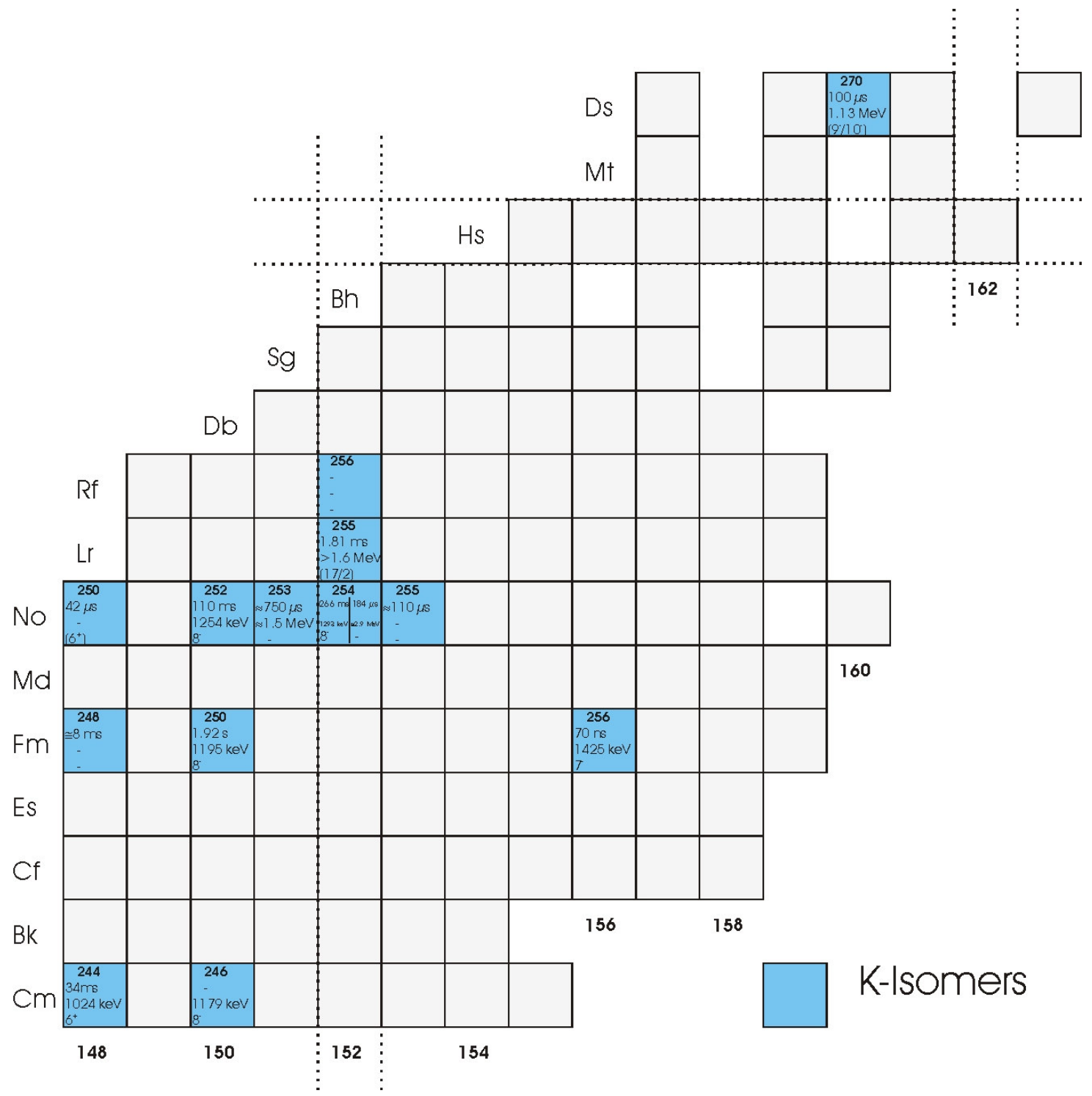

FIGURE 2. Excerpt of the chart of nuclides indicating the K-isomers observed for heavy nuclei in the region $\mathrm{Z} \square 96$ and the decay chain for the proposed reaction $64 \mathrm{Ni}+207 \mathrm{~Pb}$. Half-life, decay energy, spin and parity values are given for K-Isomers only

$\mathrm{Xu}$ et al. pointed out that high-spin $K$-isomerism has consequences for the fission barrier and $\alpha$-decay, which could lead to a higher stability and longer lifetimes of the isomeric state as compared to the ground state (g.s.) for a certain class of superheavy nuclei. They state that, "via the population of high-K states in experiments, one may be able to extend the nuclear chart further into the island of superheavy nuclei". Examples for such an isomer-g.s. lifetime inversion are ${ }^{250}$ No [27] and ${ }^{270}$ Ds [11], the heaviest nucleus for which a $K$-isomer has been observed.

\subsubsection{K-Isomers in ${ }^{252} \mathrm{No}$ and ${ }^{254} \mathrm{No}$}

For the deformed even-even isotopes ${ }^{252}$ No and ${ }^{254}$ No the ground-state band structure had been investigated in pioneering experiments at ANL and JYFL using combinations of separator and $\gamma$-ray detector arrays at the target position [18]. 
For ${ }^{254}$ No two isomeric states were observed in decay spectroscopic studies $[28,29]$. The first was placed at an excitation energy of 1293-1297 keV. Its half-life was determined with $180 \mu \mathrm{s}$. For the second isomeric state both groups estimated $\approx 2.5 \mathrm{MeV}$ but disagreed on the spin assignment. In both publications four-quasipaticle configurations are proposed for this state with $\mathrm{K}^{\pi}=16^{+}$[28] and $14^{+}$[29]. In a recent measurement at SHIP we could establish the band structure between the two isomers as well as its link to the ground state rotational band by means of $\gamma-\gamma$ coincidences between the $606 \mathrm{keV}$ line and the observed intra band transitions, and the observation of the two new transitions with $778 \mathrm{keV}$ and $856 \mathrm{keV}$ [30]. We measured a halflife for this second isomeric state of $198 \pm 13 \mu \mathrm{s}$. The resulting decay scheme is shown in Fig. 2.

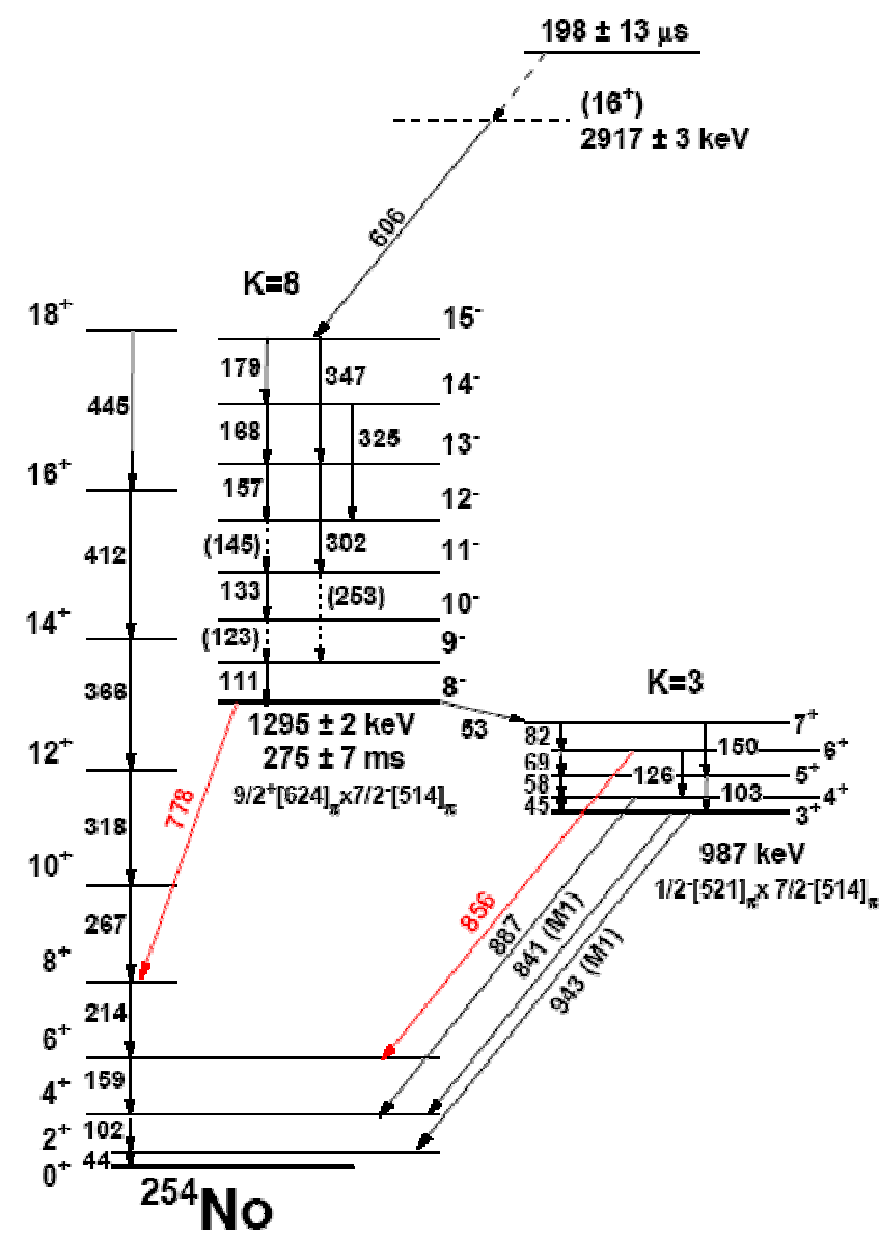

FIGURE 3. Decay scheme of ${ }^{254}$ No showing the placement and spin assignment of the two Kisomers [30].

For ${ }^{252} \mathrm{No}$ we observed for the first time a K-isomer with a half-life of $110 \pm 10 \mathrm{~ms}$ at an excitation energy of $1254 \mathrm{keV}$ [9]. Beyond the spin and parity assignment of this isomer we could establish also a detailed decay scheme including a side band below the isomeric state and its connection to the ground state rotational band.

\subsection{2 ${ }^{270} \mathrm{Ds}$ and its Decay Products}

In the experiment reported in ref. [11] we observed in the reaction ${ }^{64} \mathrm{Ni}+{ }^{207} \mathrm{~Pb}$ a total of 8 decay chains consisting of evaporation residue(ER)- $\alpha-\alpha$-sf correlations which we all attributed 
to the production of ${ }^{270}$ Ds followed by the sequential emission of two $\alpha$-particles leading two the daughter ${ }^{266} \mathrm{Hs}$ and the granddaughter ${ }^{262} \mathrm{Sg}$ which eventually decayed by spontaneous fission. During a period of 7.3 days we collected a beam dose of $1.3 \times 10^{18}$ projectiles at a beam energy of $317 \mathrm{MeV}$. At the resulting excitation energy of $14 \mathrm{MeV}$ we obtained a production cross section of $13 \pm 5 \mathrm{pb}$ which was close to the value obtained for the synthesis of ${ }^{271}$ Ds in the reaction ${ }^{64} \mathrm{Ni}+{ }^{207} \mathrm{~Pb}$. We discussed this unexpectedly high value also in terms of a possible modification of the cross section by the presence of an isomeric state.

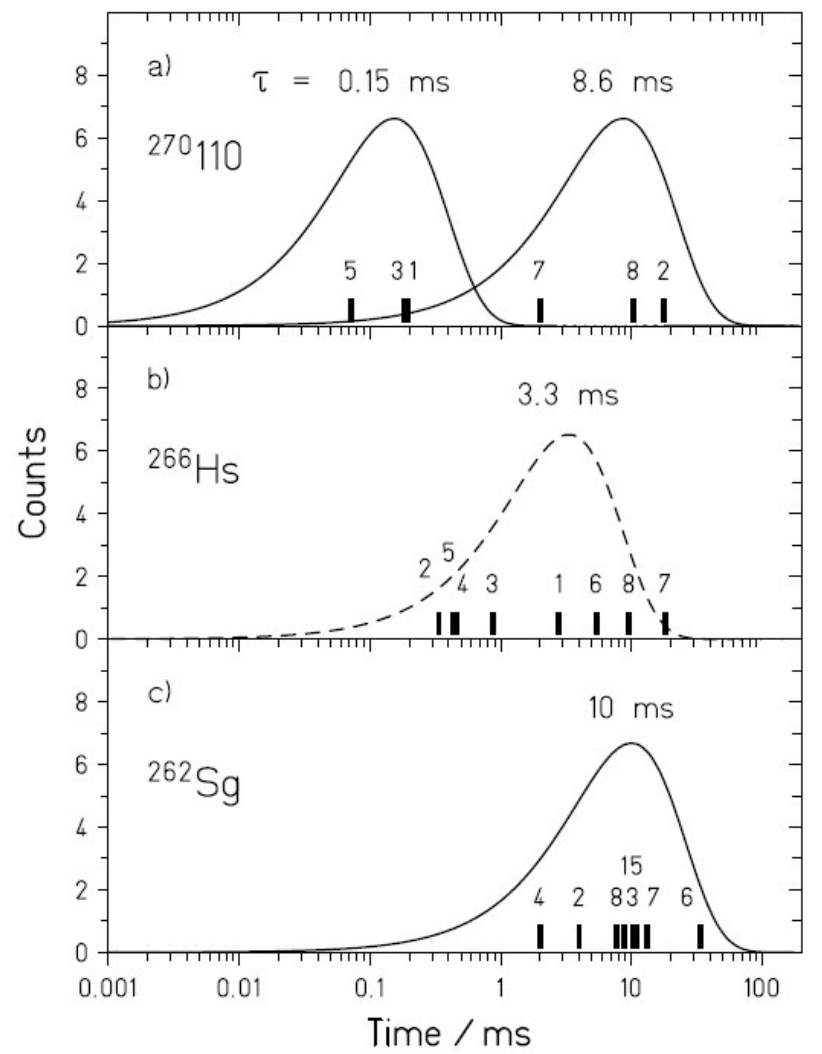

FIGURE 4. Time distribution of the subsequent $\alpha$-decays $(a, b)$ and fission events (c) measured in the reaction $64 \mathrm{Ni}+207 \mathrm{~Pb}$. The curves represent the density distribution (arbitrary ordinate scale) of counts in a radioactive decay on a logarithmic time scale with logarithmically increasing channel width [32]. The position of the curve determines the lifetime; it was adjusted to the data by a least-squares analysis. The dashed curve in plot b) indicates that the data are also compatible with two lifetimes of 0.5 and $9 \mathrm{~ms}$ (see text). The numbers from 1 to 8 assign the events to the measured decay chains (see e.g. Table 1 in ref. [11]). (Figure taken from ref. [11])

For two of the decay sequences the first $\alpha$ escaped from the detector in backward direction without generating an electronic signal in the Si detector. For the six remaining chains we observed the ${ }^{270}$ Ds $\alpha$ decay which we assigned to two groups of decay times with half-lives of $6.0_{-2.2}^{+8.2} \mathrm{~ms}$ and $100_{-40}^{+140} \mu \mathrm{s}$, respectively. The $\alpha$ decay energy of the shorter lived g.s. was $11.03 \pm 0.05 \mathrm{MeV}$, whereas for the longer lived isomer we observed $\alpha$ particles with $10.95 \pm 0.02$ $\mathrm{MeV}, 11.15 \pm 0.02 \mathrm{MeV}$ and $12.15 \pm 0.05 \mathrm{MeV}$. We could assign those six $\alpha$-decays to various decay paths which are consistent with the calculated level scheme [31]. They are illustrated in the insert in Fig. 5. We interpreted one ${ }^{270}$ Ds $\alpha$ decay (chain number 7), that belonged to the slower decay group and that was followed by a $\gamma$-ray, as the isomer decaying into an excited state of the daughter ${ }^{266} \mathrm{Hs}$. The first $\alpha$ of chain number 8 , being also part of the longer lived group, with the highest decay energy was assigned to the isomer-to-g.s. decay. From the energy 
difference of this decay and the g.s.-to-g.s. transition energy, we derived the excitation energy of the isomer of $1.13 \mathrm{MeV}$. The last slow decay, having the same $\alpha$ energy as the three faster decays is consistent with the isomer decaying by $\gamma$-emission (not observed) into the ${ }^{270 \text { g.s. }} \mathrm{Ds}$ which then decays directly into the ${ }^{266} \mathrm{Hs}$ ground state. The remaining three observed $\alpha$ particles from the shorter lived group we view as g.s.-g.s. transitions. Note: this interpretation is consistent with theoretical expectations [33] which were also confirmed by $\mathrm{Xu}$ et al. [21] who produced a similar decay scenario. However, the derived ${ }^{270 \mathrm{~m}}$ Ds excitation energy is based on just one measured $\alpha$ decay. The probability is non-zero that the transition assigned as isomerg.s. transition populates a state above the ground state instead. In that case this excitation energy value would be a lower limit. Other properties like e.g. spin and parity of the ${ }^{270} \mathrm{Ds} K$-isomer or the excitation energy of the states populated in ${ }^{266} \mathrm{Hs}$ are still missing. To settle these questions more decay data will be collected in an experiment at SHIP scheduled for spring/summer 2

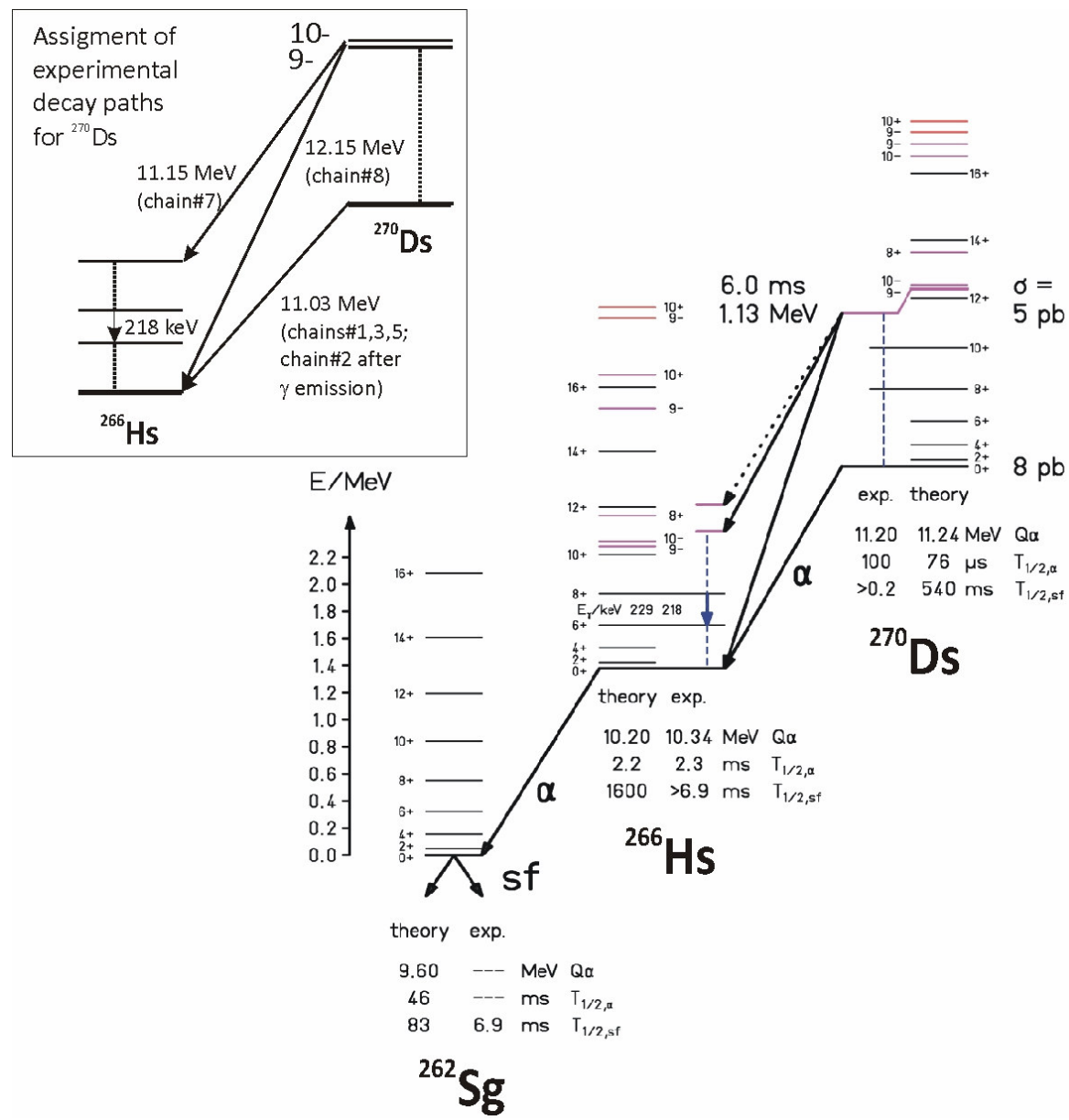

FIGURE 5. Proposed decay and level scheme for 270Ds and its decay products on the basis of our observations [11] and the predictions of Hartree-Fock-Bogoliubov calculations [23], taken from ref. [17]. The insert illustrates the decay paths for 270Ds corresponding to the six observed decay chains identified by the chain numbers from ref [11].

For the daughter ${ }^{266} \mathrm{Hs}$ the observed eight decay times were consistent with a single half-life of $2.3_{-0.6}^{+1.3} \mathrm{~ms}$ with an $\alpha$ energy of $10.18 \pm 0.02 \mathrm{MeV}$. The time distribution of the ${ }^{266} \mathrm{Hs} \alpha$ decay is relatively wide spread and we pointed out the possibility of the presence of two decay components with half-lives of $0.35_{-0.11}^{+0.28}$ and $6.3_{-2.3}^{+8.6} \mathrm{~ms}$, respectively. This would be in line with 
the expectations from the theoretical predictions of the existence of $K$ isomers in the whole region of deformed heavy and superheavy elements. Following our decay assignment above, one out of six ${ }^{270}$ Ds $\alpha$ decays (chain number 7) populates an excited state in ${ }^{266} \mathrm{Hs}$. Therefore, also here larger statistics is mandatory.

The last member of the decay chain decays by fission with a half-life of $6.9_{-1.8}^{+3.8} \mathrm{~ms}$ and a total kinetic energy of the fission fragments of $222 \pm 10 \mathrm{MeV}$. The time distributions are shown in Fig. 4. From the decay data which we observed in our first measurement, and the predictions of Hartree-Fock-Bogoliubov model calculations [33] we constructed a level scheme which is shown in Fig. 5. There we also marked by an arrow the $8^{+}$to $6^{+}$transition in the g.s. rotational band of ${ }^{266} \mathrm{Hs}$. It is predicted to have a transition energy of $229 \mathrm{keV}$, a value which is close to the $218 \mathrm{keV}$ we measured for a $\gamma$-ray in coincidence with the $11.15 \mathrm{MeV} \alpha$ particle of the longer lived ${ }^{270}$ Ds decay (decay chain number 7) in our first measurement.

The expected $\alpha$-sf branching ratios for ${ }^{270} \mathrm{Ds}$ and ${ }^{266} \mathrm{Hs}$ are relatively low. In ref. [11] we estimated "optimistic" upper limits with 0.2 and $1.4 \%$, respectively. A search for those sfbranches is very challenging and probably only marginally in reach of this proposal. For ${ }^{262} \mathrm{Sg}$ our measured limit for an $\alpha$-branch of $22 \%$ was recently improved be K.E. Gregorich et al. [34] who obtained a limit value of $16 \%$. This is very close to the prediction of $15 \%$ [11], and with a slightly increased number of observed events it should be possible to observe this decay mode.

\section{Outlook: Towards the Island of Stability}

Low cross sections, the advances in nuclear structure investigations, reaction mechanism studies, chemistry and SHE synthesis experiments with a steadily increasing demand for higher beam intensities, more sensitive and more sophisticated detection set-ups and new methods determine the road map for future SHE investigations. Intensity increase is one of the major issues in this context. The presently pursued upgrade of the UNILAC accelerator at GSI, consisting of a new $28 \mathrm{GHZ}$ ECR source and a new RFQ injector providing an order of magnitude higher beam current, is only a first step towards a dedicated continuous wave (CW) accelerator which would increase the beam intensity by a factor of four already by extending the $25 \%$ UNILAC duty cycle to $100 \%$. Additional increases due to the advanced accelerator technology can be expected. Improvement of the detection system in terms of higher efficiencies is as mandatory as the employment of additional measurement parameters. Mass determination by an adequate spectrometer, in contrast to precision mass measurements with a trap, would be extremely helpful for a final confirmation of the unconnected chains obtained in the ${ }^{48} \mathrm{Ca}$ induced reactions on actinide targets.

In conclusion, the roadmap towards spherical shell stabilized nuclei is laid out. The challenges are obvious. It is up to us to take them on.

\section{References}

[1] H. Meldner, Proc. of the Int .Symp. on Nuclides far off the Stability Line, Lysekil, Sweden, Agust 21-27, 1966, edited by W. Forsling, C.J. Herrlander, and H. Ryde, Ark. Fys. 36, 593.

[2] Yu.Ts. Oganessian, J. Phys. G34, R165 (2007).

[3] S. Hofmann and G. Münzenberg, Rev. Mod. Phys. 72, 733 (2000).

[4] R. Smolanczuk et al., Phys. Rev. C 52, 1871 (1995).

[5] K. Morita et al., J. Phys. Soc. Jpn. 73, 2593 (2004). 
[6] S. Hofmann et al., Eur. Phys. J. A 32, 251 (2007).

[7] L. Stavsetra et al., Phys. Rev. Lett. 103, 132502 (2009).

[8] C. Düllmann et al., submitted to PRL.

[9] B. Sulignano et al., Eur. Phys. J. A 33, 327 (2007)

[10] R.D. Herzberg et al., Nature 442, 896 (2006).

[11] S. Hofmann et al., Eur. Phys. J. A 10, 5 (2001).

[12] M. Block et al., Nature 463, 785 (2010).

[13] Yu.Ts. Oganessian et al., Phys. Rev. C 70, 064609 (2004).

[14] Yu.Ts. Oganessian et al., Phys. Rev. C 74, 044602 (2006).

[15] R. Eichler et al., Nature 447, 72 (2007).

[16] M.Leino and F.P. Heßberger, Ann.Rev.Nucl.Part.Sci. 54, 175 (2004).

[17] L.-L. Andersson et al. submitted to Nucl. Instr. and Meth. A.

[18] R.-D. Herzberg and P.T. Greenlees, Prog. Part. Nuc. Phys. 61, 674 (2008).

[19] P.M. Walker and G.G. Dracoulis, Nature 399, 35 (1999).

[20] K.E.G. Löbner, Phys. Lett. B 26, 369 (1968).

[21] F.R. Xu et al., Phys. Rev. Lett. 92, 252501 (2004).

[22] F.P. Heßberger et al., Eur. Phys. J. A 30, 561 (2006).

[23] F.P. Heßberger et al., Eur. Phys. J. A 29, 165 (2006).

[24] F.P. Heßberger et al., Phys. At. Nuc. 8, 1445 (2007)

[25] K. Hauschild et al., Phys.Rev. C 78, 021302 (2008).

[26] S. Antalic et al., Eur. Phys. J. A 38, 219 (2008).

[27] D. Peterson et al., Phys. Rev. C 74, 014316 (2006).

[28] R.-D. Herzberg et al., Nature 442, 896 (2006).

[29] S.K. Tandel et al., Phys. Rev. Lett. 97, 082502 (2006).

[30] F.P. Heßberger et al., accepted for publication at Eur. Phys. J. A.

[31] S. Hofmann, Jour. Nucl. and Radiochem. Sc. 4, R1 (2003).

[32] K.H. Schmidt et al., Z. Phys. A 316, 19 (1984).

[33] S. Cwiok, W. Nazarewicz and P.H. Heenen, Phys. Rev. Lett. 83, 1108 (1999).

[34] K.E. Gregorich et al., Phys. Rev. C 74, 044611 (2006). 